\title{
Inactivation of SARS-CoV-2 Laboratory Specimens
}

\author{
Elaine Haddock, ${ }^{1}$ Friederike Feldmann, ${ }^{2}$ W. Lesley Shupert, ${ }^{1}$ and Heinz Feldmann ${ }^{1 \star}$ \\ ${ }^{1}$ Laboratory of Virology, Division of Intramural Research, National Institute of Allergy and Infectious Diseases, National Institutes of Health, \\ Hamilton, Montana; ${ }^{2}$ Rocky Mountain Veterinary Branch, Division of Intramural Research, National Institute of Allergy and Infectious Diseases, \\ National Institutes of Health, Hamilton, Montana
}

\begin{abstract}
The burden on diagnostic and research laboratories to provide reliable inactivation for biological specimens to allow for safe downstream processing is high during the coronavirus disease 2019 (COVID-19) pandemic. We provide safety data regarding commonly used chemical and physical inactivation procedures that verify their effectiveness against severe acute respiratory syndrome coronavirus 2 (SARS-CoV-2).
\end{abstract}

Severe acute respiratory syndrome coronavirus 2 (SARSCoV-2) is the cause of the current coronavirus disease 2019 (COVID-19) pandemic. $^{1-3}$ There is a high demand for safe sample handling to perform tests on in vitro and in vivo material in research and diagnostic laboratories worldwide. SARS-CoV-2 is considered a risk group 3 pathogen, and safety at workplaces is of the highest priority. ${ }^{4}$ SARS-CoV-2 is an enveloped, single-strand, positive-sense RNA virus with a genome size of approximately $30 \mathrm{~kb} .^{2,3,5}$ Its biological, biochemical, and physical features make the virus sensitive to chemical and physical inactivation procedures. We evaluated commonly used inactivation procedures to generate safe material for downstream genome, protein, immune response, and histopathology analyses.

All infectious work was performed under high biocontainment conditions at the Rocky Mountain Laboratories of the National Institute of Allergy and Infectious Diseases (NIAID) according to standard operating protocols (SOPS) approved by the Institutional Biosafety Committee (IBC). For our studies, we used the SARS-CoV-2 isolate nCoV-WA12020 (MN985325.1; kindly provided by the Centers for Disease Control and Prevention). ${ }^{6}$ SARS-CoV-2 replication in cell culture causes a cytopathic effect (CPE), thus allowing for a simple readout parameter. SARS-CoV-2 stocks were grown in VeroE6 cells and titrated using a tissue culture infectious dose $50 \%\left(\mathrm{TCID}_{50}\right)$ assay. ${ }^{7}$ The $\mathrm{TCID}_{50}$ was calculated via the Reed-Muench formula to a concentration of $4 \times 10^{6} \mathrm{TCID}_{50} /$ $\mathrm{mL} .{ }^{8}$ Cells were produced by infecting VeroE6 cells at a multiplicity of infection of 0.01 SARS-CoV-2. Cells were harvested in Dulbecco's phosphate-buffered saline (DPBS) at CPE of approximately $75 \%$, counted, and frozen $\left(-80^{\circ} \mathrm{C}\right)$ in aliquots of $2 \times 10^{6}, 5 \times 10^{6}$, and $2 \times 10^{7}$ cells $/ \mathrm{mL}$. SARS-CoV-2-infected lung tissue $\left(\approx 1 \times 10^{10} \mathrm{TCID}_{50} / \mathrm{g}\right)$ was obtained from a previous Syrian hamster study approved by the Institutional Animal Care and Use Committee. ${ }^{9}$

We tested the physical and chemical inactivation of virus stocks as well as chemical inactivation of infected cells and tissue. Triplicate samples were dialyzed with an 8- to 10-kDa molecular weight cutoff (Repligen Corporation, Waltham, MA) using DPBS over a stir plate at $4^{\circ} \mathrm{C}$ (> 500-fold exchange volumes, five changes during 32-48 hours) or run over a detergent removal column (DetergentOUT GBS10-5000 columns; G-Biosciences, St. Louis, MO). DPBS and noninfected

*Address correspondence to Heinz Feldmann, Rocky Mountain Laboratories, 903 South $4^{\text {th }}$ Street, Hamilton, MT 59840. E-mail: feldmannh@niaid.nih.gov
VeroE6 cells and hamster lung tissues served as negative controls. Untreated virus stocks and SARS-CoV-2-infected VeroE6 cells and hamster lung tissue were used as positive controls. All samples were brought to a final volume of $3 \mathrm{ml}$ and equally divided to infect VeroE6 cells (80\% confluency) in triplicate for a total of 9 flasks per sample type. Cells were incubated at $37^{\circ} \mathrm{C}$ for 7 days and monitored regularly for CPE. Three days after initial infection, samples were passaged by transferring $1 \mathrm{~mL}$ supernatant each to a new flask of VeroE6 cells ( $80 \%$ confluency). Additionally, we tested extracted RNA with or without transfection reagents to assess RNA infectivity. The results are summarized in Table 1, and detailed protocols are provided as Supplementary Material.

\section{IRRADIATION}

Inactivation by irradiation is the preferred method for specimens used for a variety of serological, immunological, and biochemical assays, for which authentic protein and particle structure are critical. We previously determined that a dose of 1.0 Megarad (10,000 Gray) completely inactivated SARS-CoV-1, strain Tor 2 (10). To determine a breakthrough dose for inactivation, we treated $1 \times 10^{6} \mathrm{TCID}_{50}$ of SARS-CoV2 with 0, 0.2, 0.4 0.6, 0.8, and 1.0 Megarad. We used a JL Shepherd Model 484R irradiator (JL Shepherd and Associates, San Fernando, CA) using a cobalt-60 source as previously described. ${ }^{10}$ Irradiation was performed on dry ice and samples were co-located with lithium fluoride film dosimeters on a rotating platform to assure homogeneous irradiation and confirm the absorbed doses. VeroE6 cells were infected in triplicate with the treated samples and monitored for CPE. As with SARS-CoV-1, only the dose of 1.0 Megarad completely inactivated SARS-CoV-2 (Table 1).

\section{CHAOTROPIC REAGENTS}

For nucleic acid extraction, we used the guanidinium isothiocyanate buffers AVL and RLT (Qiagen, Hilden, Germany) as well as TRIzol (Life Technologies, Carlsbad, CA), as recommended by the manufacturers. AVL was mixed with stock virus at a ratio of 1:5 (sample:total) and incubated for 10 minutes at room temperature, followed by transfer to $560 \mu \mathrm{L}$ of $100 \%$ ethanol for 20 minutes at room temperature (Table 1). Infected cells $\left(5 \times 10^{6}\right)$ were resuspended and incubated in RLT for 10 minutes, followed by the addition of $600 \mu \mathrm{L}$ of $70 \%$ ethanol for an incubation period of 20 minutes. Infected lung tissue was homogenized in RLT with a stainless-steel bead 


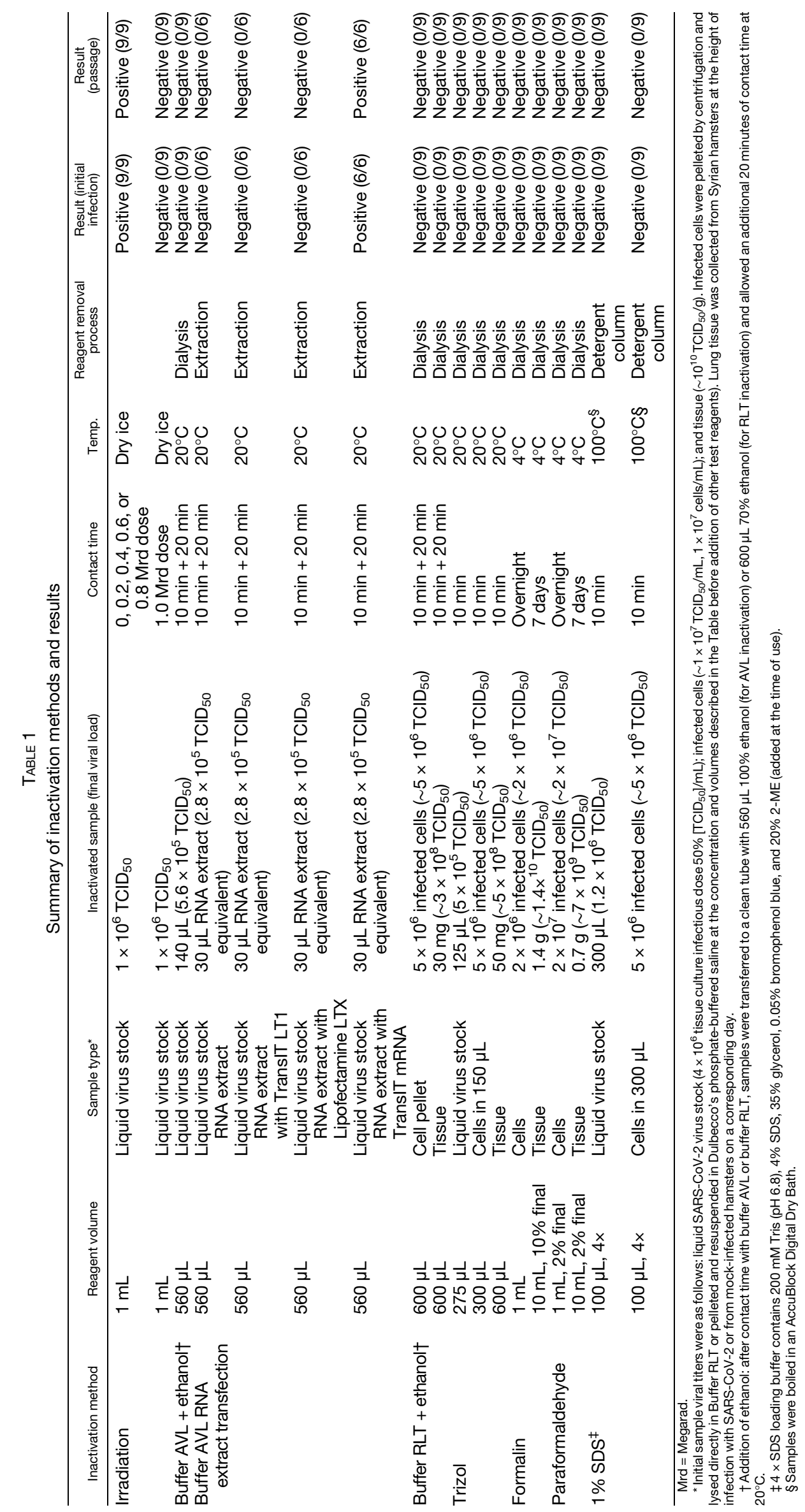


(10 minutes at $30 \mathrm{~Hz}$ ). A soluble aliquot (equivalent to $\approx 30 \mathrm{mg}$ ) was transferred to a new tube and fresh RLT was added to a volume of $600 \mu \mathrm{l}$, followed by the addition of $70 \%$ ethanol $(600 \mu \mathrm{L})$ for 20 minutes. TRIzol was mixed with stock virus at a ratio of 1:3.2 (sample:total) and incubated for 10 minutes at room temperature (Table 1$)$. Infected cells $\left(5 \times 10^{6}\right)$ were resuspended and treated with TRIzol (1:3 sample:total). Infected lung samples were homogenized, and an aliquot of tissue homogenate $(\approx 50 \mathrm{mg})$ was transferred to a new tube and fresh TRIzol was added. After immediate dialysis, VeroE6 cells were inoculated with the treated material and monitored for CPE. In all cases, no CPE was observed, indicating complete inactivation of SARS-CoV-2 by AVL, RLT, and TRIzol (Table 1).

\section{RNA INFECTIVITY}

The single-strand, positive-sense RNA genome of coronaviruses may start a replication cycle on entry into cells, subsequently generating infectious virus. Therefore, we performed direct infectivity testing of extracted RNA in water on VeroE6 cells. RNA was simply added to the cell monolayer or transfected with the reagents Lipofectamine LTX (Invitrogen, Carlsbad, CA), TransIT LT1 (Mirus Bio, Madison, WI), or TransIT-mRNA (Mirus Bio) according to the manufacturers' recommendations. Although TransIT LT1 is designed primarily for DNA transfection and unlikely to aid the uptake of the SARS-CoV-2 RNA genome, Lipofectamine LTX supports transfection of both RNA and DNA components, and TransITmRNA is specifically designed for RNA transfection. Transfection mixtures containing $30 \mu \mathrm{L}$ RNA (one-half the elution volume) were tested in duplicate, and each sample was evenly split across three wells of a six-well plate of VeroE6 cells at $80 \%$ confluency. No CPE was observed by viral RNA alone or transfection with reagents other than TransIT-mRNA, indicating that SARS-CoV-2 genomic RNA is noninfectious under these conditions (Table 1).

\section{FIXATIVES}

Formalin and paraformaldehyde are commonly used to fix cells or tissues for histologic or microscopic analyses. Infected cells $\left(2 \times 10^{6}\right)$ were diluted in $1 \mathrm{~mL} 10 \%$ neutral-buffered formalin (Leica Biosystems, Wetzlar, Germany) or $\left(2 \times 10^{7}\right) 2 \%$ paraformaldehyde (Electron Microscopy Services, Hatfield, PA) overnight at $4^{\circ} \mathrm{C}$. Samples were pelleted by centrifugation and washed with DPBS in a minimum of three cycles to remove fixative and used to infect VeroE6 cells. Monitoring of cells revealed the absence of CPE, indicating complete inactivation of SARS-CoV-2 (Table 1).

Infected hamster tissue was incubated in $10 \mathrm{~mL}$ of $10 \%$ neutral-buffered formalin (full lungs) or $2 \%$ paraformaldehyde (half lung) for a period of 7 days at $4^{\circ} \mathrm{C}$. Subsequently, a small section of tissue $(\approx 150 \mathrm{mg})$ was dissected and homogenized in DPBS. After a minimum of three cycles of pelleting and washing with DPBS, samples were used to infect VeroE6 cells. Monitoring of cells confirmed the absence of CPE, indicating complete inactivation of SARS-CoV-2 (Table 1).

\section{DETERGENTS}

Detergent treatment under heat is often used for protein analysis. Aliquots of virus stock $(300 \mu \mathrm{L})$ or infected cells $\left(5 \times 10^{6}\right.$ in $\left.300 \mu \mathrm{L}\right)$ were diluted in $100 \mu \mathrm{L} 4 \times$ loading buffer ( $1 \%$ sodium dodecyl sulfate [SDS] final) and boiled at $100^{\circ} \mathrm{C}$ for 10 minutes. Detergent was immediately removed through DetergentOUT columns (G-Biosciences). Treated samples were tested for inactivation on VeroE6 cells. Although titers were not measured from control samples after this treatment, CPE via the positive samples was not delayed, indicating that a significant reduction in virus because of treatment alone was unlikely. In test samples, no CPE was noticed, indicating complete inactivation of SARS-CoV-2 (Table 1).

\section{CONCLUSIONS}

The study verified the safe use of common chemical and physical treatment procedures for the complete inactivation of SARS-CoV-2 in distinct specimen types (Table 1). Additionally, we have shown that SARS-CoV-2 genomic RNA alone is unlikely to spontaneously generate virus from introduction to cells. The inactivation procedures are likely to apply to all coronaviruses because of the similar biological, biochemical, and biophysical features within the virus family. These data may provide guidance for IBCs to evaluate SOPs for the inactivation of SARS-CoV-2 containing biological specimens. The well-defined results may also assist in the improvement and approval of SOPs for inactivation without the need to verify inactivation for individual samples, which is unfeasible with the current diagnostic and research operations.

Received February 24, 2021. Accepted for publication April 8, 2021. Published online April 20, 2021.

Acknowledgments: The authors acknowledge the Viral Special Pathogens Branch of the Centers for Disease Control and Prevention, Atlanta, GA, USA, for providing SARS-CoV-2. The American Society of Tropical Medicine and Hygiene has waived the Open Access fee for this article due to the ongoing COVID-19 pandemic.

Financial support: Funding for this study was provided by the Intramural Research Program, National Institute of Allergy and Infectious Diseases, National Institutes of Health.

Authors' addresses: Elaine Haddock, W. Lesley Shupert, and Heinz Feldmann, Laboratory of Virology, Division of Intramural Research, National Institute of Allergy and Infectious Diseases, National Institutes of Health, Hamilton, MT, E-mails: elaine.haddock@nih.gov, wlshupert@niaid.nih.gov, and feldmannh@niaid.nih.gov. Friederike Feldmann, Rocky Mountain Veterinary Branch, Division of Intramural Research, National Institute of Allergy and Infectious Diseases, National Institutes of Health, Hamilton, MT, E-mail: feldmannfe@ niaid.nih.gov.

This is an open-access article distributed under the terms of the Creative Commons Attribution (CC-BY) License, which permits unrestricted use, distribution, and reproduction in any medium, provided the original author and source are credited.

\section{REFERENCES}

1. World Health Organization, 2021. Weekly Epidemiological Update-16 February 2021. Available at: https://www. who.int/publications $/ \mathrm{m} /$ item/weekly-epidemiological-update16-february-2021.

2. Ortega MA et al., 2021. An integrative look at SARS-CoV-2 (Review). Int J Mol Med 47: 415-434.

3. Gorbalenya AE et al., Coronaviridae Study Group of the International Committee on Taxonomy of Viruses, 2020. The species severe acute respiratory syndrome-related coronavirus: classifying 2019-nCoV and naming it SARS-CoV-2. Nat Microbiol 5: 536-544. 
4. World Health Organization, 2020. Laboratory Biosafety Guidance Related to the Novel Coronavirus (2019-nCoV Interim Guidance12 February. Available at: https://www.who.int/docs/default-source/ coronaviruse/laboratory-biosafety-novel-coronavirus-version1-1.pdf?sfvrsn=912a9847_2.

5. V'kovski P, Kratzel A, Steiner S, Stalder H, Thiel V, 2021. Coronavirus biology and replication: implications for SARS-CoV-2. Nat Rev Microbiol 19: 155-170.

6. Harcourt $\mathrm{J}$ et al., 2020. severe acute respiratory syndrome coronavirus 2 from patient with 2019 novel coronavirus disease, United States. Emerg Infect Dis 26: 1266-1273.
7. Munster VJ et al., 2020. Respiratory disease in rhesus macaques inoculated with SARS-CoV-2. Nature 585: 268-272.

8. Reed LJ, Muench H, 1938. A simple method of estimating fifty percent endpoints. Am J Hyg 27: 493-497.

9. Rosenke K et al., 2020. Defining the Syrian hamster as a highly susceptible preclinical model for SARS-CoV-2 infection. Emerg Microbes Infect 9: 2673-2684.

10. Feldmann F, Shupert WL, Haddock E, Twardoski B, Feldmann H, 2019. Gamma irradiation as an effective method for inactivation of emerging viral pathogens. Am J Trop Med Hyg 100: 1275-1277. 